\title{
On the behavior of single-surface nuclear wavefunctions in the vicinity of the conical intersection for an $\mathrm{X}_{3}$ system
}

\author{
A.J.C. Varandas * , Z.R. Xu \\ Departamento de Química, Universidade de Coimbra, 3049 Coimbra Codex, Portugal \\ Received 2 February 1999; in final form 1 November 1999
}

\begin{abstract}
The behavior of single-surface nuclear wavefunctions in the vicinity of the conical intersection at the equilateral triangle conformations of homonuclear triatomic systems is examined analytically by using spherical coordinates which are obtained from the usual $D_{3 h}$ normal mode ones. They are described by a set of three quantum numbers and shown to approach zero at the conical intersection, irrespective of whether the so-called geometric phase effects are taken into consideration. (C) 2000 Elsevier Science B.V. All rights reserved.
\end{abstract}

\section{Introduction}

In recent years, there has been considerable interest in molecular vibronic dynamics which requires the consideration of two or more Born-Oppenheimer (BO) potential energy surfaces (PES) [1-4]. In particular, recent interest has focused on the so-called conical intersections. The simplest systems exhibiting such an intersection are those formed by three identical ${ }^{2} \mathrm{~S}$ atoms, i.e., $\mathrm{H}_{3}$ [5,6], $\mathrm{Li}_{3}$ [7-9], $\mathrm{Na}_{3}$ [10], etcetera. For these systems, symmetry considerations force the conical intersection to occur at $D_{3 h}$ geometries; the behavior of the corresponding $\mathrm{BO}$ energies and the derivative coupling has been examined by Mead and co-workers [2,4]. In particular, Mead [2] has proved that single-surface nuclear wavefunctions for such a system must approach zero at the conical intersection [2], a finding which may

\footnotetext{
* Corresponding author. Fax: +351-39-27703; e-mail: varandas@qtvsl.uc.pt
}

have implications on the behavior of the derivative coupling terms at the conical intersection [2]. However, Mead's demonstration is based on a two-dimensional (2D) model analysis, a limitation which has been removed in the present work while using a relatively straightforward formalism.

Related to conical intersections are subtle complications arising in the adiabatic treatment of the nuclear motion, which are known as geometric phase (GP) effects [11] (also often called Bohm-Aharonov effects [2] or Berry's phase effects [12,2]). Such complications stem in molecular dynamics from two topological theorems due to Longuet-Higgins [13] which state (1) that on going once around any closed path on the (adiabatic) PES that contains the conical intersection the electronic wavefunction changes sign and (2) that if a real adiabatic electronic wavefunction changes sign when a polyatomic traverses a one-dimensional closed loop on a $2 \mathrm{D}$ surface in the $(3 N-6)$-dimensional nuclear configuration space, then the corresponding electronic state must become 
discontinuous and degenerate with another one at an odd number of points lying on that surface and within that loop. Indeed, ab initio electronic structure calculations have shown such theorems to hold, e.g., for LiNaK [14], a system which has no permutational symmetry. Since the total electronuclear wavefunction must be well behaved, one has therefore to take GP effects into account when carrying out singlesurface calculations. Only in this case will the results satisfy the proper boundary conditions and hence the minimum requirement for comparison with experiment. Such an approach has been extensively explored recently, in particular by Kendrick and Pack [15] who have introduced the so-called generalized BO method (for further discussion on this topic, see Refs. [16,17]). Billing and Markovic [18] carried out similar calculations but using hyperspherical coordinates to include the GP effects; for $\mathrm{X}_{3}$ molecules having a single $D_{3 h}$ conical intersection, GP effects concern only the $\phi$ hyperangle. A similar approach has been advocated by us in previous studies [6,8,9,1].

In this Letter, we discuss in detail the behavior of the nuclear wavefunctions for $\mathrm{X}_{3}$ systems in the vicinity of the $D_{3 h}$ conical intersection and compare the results with those reported previously by Mead [2] from his 2D model system. Section 2 presents a brief discussion on the reduction of the coupled equations, while the behavior of the nuclear wavefunctions in the vicinity of the conical interection will be discussed in Section 3. Conclusions are in Section 4.

\section{Coupled equations and nonadiabatic coupling}

Consider the dynamics of an $\mathrm{X}_{3}$ system in the vicinity of the $D_{3 h}$ crossing seam. The conventional coordinates used for this purpose are the normal coordinates $\left(Q_{x}, Q_{y}, Q_{z}\right)[19,20]$ illustrated in Fig. 1 , or the corresponding spherical coordinates $(\rho, \theta, \varphi)$ which are related to them by

$Q_{x}=\rho \sin \theta \cos \varphi$,

$Q_{y}=\rho \sin \theta \sin \varphi$,

$Q_{z}=\rho \cos \theta$.
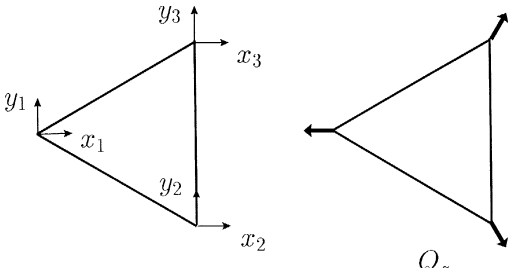

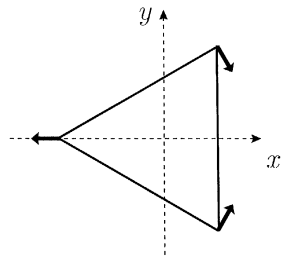

$Q_{x}$

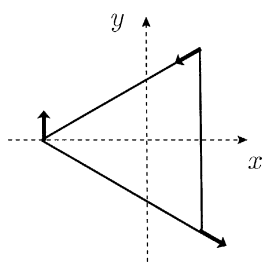

$Q_{y}$
Fig. 1. Normal coordinates for a triatomic molecule near equilateral triangle configurations.

Clearly, $Q_{x}$ and $Q_{y}$ form a basis for the $E$ representation in the $D_{3 h}$ symmetry point group, while $Q_{z}$ spans the corresponding $A_{1}$ representation. Overall translation and rotation are assumed to have been removed.

Following the usual procedure, the total molecular wavefunction can be expanded as

$\Omega(\mathbf{Q}, \mathbf{r})=\sum_{n} \Psi_{n}(\mathbf{Q}) \chi_{n}(\mathbf{r} ; \mathbf{Q})$

where $\mathbf{r}$ and $\mathbf{Q}$ denote the sets of electronic and nuclear coordinates, respectively, and $\chi_{n}(\mathbf{r} ; \mathbf{Q})$ is the $n$-th member of the orthonormal set of eigenfunctions of the electronic Hamiltonian operator $\hat{H}_{e}(\mathbf{r}, \mathbf{Q})$; note that such functions form a complete set in $\mathbf{r}$ for any value of the coordinates $\mathbf{Q}$ on which they have a parametric dependence.

Substitution of Eq. (2) into the full time-independent Schrödinger equation (relativistic effects are ignored) yields

$$
\begin{aligned}
& \left\{-\frac{\hbar^{2}}{2 \mu}\left[\nabla^{2}+2 \mathbf{F}(\mathbf{Q}) \cdot \nabla+\mathbf{G}(\mathbf{Q})\right]+\mathbf{V}(\mathbf{Q})\right\} \\
& \mathbf{\Psi}(\mathbf{Q})=E \mathbf{\Psi}(\mathbf{Q})
\end{aligned}
$$

where $\mu$ is the reduced mass of the three-particle system, $\boldsymbol{\Psi}(\mathbf{Q})$ denotes a column vector whose components are the nuclear wavefunctions $\Psi_{n}(\mathbf{Q})$, and 
the matrix elements of $\mathbf{F}(\mathbf{Q}), \mathbf{G}(\mathbf{Q})$ and $\mathbf{V}(\mathbf{Q})$ assume the form

$F_{j k}(\mathbf{Q})=\left\langle\chi_{j}(\mathbf{r} ; \mathbf{Q}) \mid \nabla \chi_{k}(\mathbf{r} ; \mathbf{Q})\right\rangle$,

$G_{j k}(\mathbf{Q})=\left\langle\chi_{j}(\mathbf{r} ; \mathbf{Q}) \mid \nabla^{2} \chi_{k}(\mathbf{r} ; \mathbf{Q})\right\rangle$,

$V_{j k}(\mathbf{Q})=\left\langle\chi_{j}(\mathbf{r} ; \mathbf{Q})\left|\hat{H}_{e}\right| \chi_{k}(\mathbf{r} ; \mathbf{Q})\right\rangle$,

with the integration referring only to the electronic degrees of freedom. Note that the set $\chi_{n}(\mathbf{r} ; \mathbf{Q})$ may be chosen for convenience. For example, in the adiabatic representation, they are taken as eigenfunctions of $\hat{H}_{e}$ for every $\mathbf{Q}$, such that the coupling between the different components of $\Psi_{n}(\mathbf{Q})$ is confined to $\mathbf{F}(\mathbf{Q})$ and $\mathbf{G}(\mathbf{Q})$; this is often referred to as the $\mathrm{BO}$ adiabatic approximation. Alternatively, the system of coupled differential equations can be simplified through the use of a new set of electronic wavefunctions $\left\{\phi_{m}(\mathbf{r} ; \mathbf{Q})\right\}$, which are obtained from a unitary transformation of the adiabatic set $\left\{\chi_{n}(\mathbf{r} ; \mathbf{Q})\right\}$ according to

$\phi_{m}(\mathbf{r} ; \mathbf{Q})=\sum_{n} a_{m n}(\mathbf{Q}) \chi_{n}(\mathbf{r} ; \mathbf{Q})$.

In principle, the coefficients $a_{m n}(\mathbf{Q})$ can be chosen by imposing

$$
\left\langle\phi_{m}(\mathbf{r} ; \mathbf{Q}) \mid \nabla \phi_{n}(\mathbf{r} ; \mathbf{Q})\right\rangle=0
$$

for all $m$ and $n$ at any $\mathbf{Q}$. This so-called adiabaticdiabatic transformation [21] leads to the system of linear differential equations

$\nabla a_{m n}(\mathbf{Q})+\sum_{s} a_{m s}(\mathbf{Q})\left\langle\chi_{n}(\mathbf{r} ; \mathbf{Q}) \mid \nabla \chi_{s}(\mathbf{r} ; \mathbf{Q})\right\rangle=0$,

the solution of which, as well as the conditions for having such a solution, has been a matter of discussion over the years [22,2,23-25]. In the following, we will be concerned with just two electronic states, $\chi_{+}(\mathbf{r} ; \mathbf{Q})$ and $\chi_{-}(\mathbf{r} ; \mathbf{Q})$, where $+(-)$ stands for the upper (lower) sheet of the BO potential energy hypersurfaces, which are degenerate for $\rho=0$.

Using the coordinates $\left(Q_{z}, \rho, \varphi\right)$, one then has [7]

$\nabla=\frac{\partial}{\partial Q_{z}}+(\cos \varphi+\sin \varphi) \frac{\partial}{\partial \rho}+\frac{\cos \varphi-\sin \varphi}{\rho} \frac{\partial}{\partial \varphi}$ which, in the vicinity of the conical intersection, may be reduced to the simplified form

$$
\nabla=\frac{\cos \varphi-\sin \varphi}{\rho} \frac{\partial}{\partial \varphi} \text {. }
$$

As pointed out long ago by Longuet-Higgins [26], for $\rho \rightarrow 0$, the electronic wavefunctions satisfy

$\frac{\partial}{\partial \varphi} \chi_{ \pm}(\mathbf{r} ; \mathbf{Q})=\mp \frac{1}{2} \chi_{\mp}(\mathbf{r} ; \mathbf{Q})$

which, after use of Eqs. (11) and (12) into Eq. (9), leads to

$$
\frac{\partial}{\partial \varphi} a_{m \pm}(\mathbf{Q})=\mp \frac{1}{2} a_{m \mp}(\mathbf{Q}), \quad m=x, y,
$$

where $x$ and $y$ refer to $\phi_{x}$ and $\phi_{y}$ in Eq. (7). Thus, from Eqs. (7) and (13), a suitable choice of real orthogonal solutions is

$$
\begin{aligned}
& {\left[\begin{array}{l}
\phi_{x}(\mathbf{r} ; \mathbf{Q}) \\
\phi_{y}(\mathbf{r} ; \mathbf{Q})
\end{array}\right]} \\
& \quad=\left[\begin{array}{cc}
\cos (\varphi / 2) & \sin (\varphi / 2) \\
-\sin (\varphi / 2) & \cos (\varphi / 2)
\end{array}\right]\left[\begin{array}{c}
\chi_{+}(\mathbf{r} ; \mathbf{Q}) \\
\chi_{-}(\mathbf{r} ; \mathbf{Q})
\end{array}\right] .
\end{aligned}
$$

Note that the adiabatic eigenfunctions in Eq. (14) are not single-valued functions of the nuclear coordinates. However, as pointed out by Mead and Truhlar $[27,28]$, an appropriate choice of single-valued eigenfunctions is

$\tilde{\chi}_{+}=\chi_{+} \exp [\mathrm{i} f(\mathbf{Q})], \quad \tilde{\chi}_{-}=\chi_{-} \exp [\mathrm{i} f(\mathbf{Q})]$

where the function $f(\mathbf{Q})$ may not be single-valued; indeed, it may increase by a multiple of $2 \pi$ on traversing a closed path around the conical intersection. Thus, the adiabatic electronic wavefunctions can be made continuous and single-valued simply by multiplying them by a phase factor. One gets $[27,28]$

$\mathbf{F}(\mathbf{Q}) \rightarrow \mathbf{F}(\mathbf{Q})+\mathrm{i} \nabla f(\mathbf{Q})$,

$\mathbf{G}(\mathbf{Q}) \rightarrow \mathbf{G}(\mathbf{Q})+2 \mathrm{i} \mathbf{F}(\mathbf{Q}) \cdot \boldsymbol{\nabla} f+\mathrm{i} \nabla^{2} f-(\nabla f)^{2}$,

which is equivalent to the transformation in Eq. (3) $\nabla \rightarrow \nabla+\mathrm{i} \nabla f(\mathbf{Q})$.

This implies that the solution of the resulting Schrödinger equation can be obtained by multiplying the original nuclear wavefunctions by a phase factor 
$\exp [-\mathrm{i} f(\mathbf{Q})]$ such as to leave the total wavefunction unchanged. A convenient choice for $\mathrm{X}_{3}$ molecules with a $D_{3 h}$ conical intersection is $f(\mathbf{Q})=j \varphi / 2$, where $j$ is an integer. This approach has been shown to be particularly convenient when hyperspherical coordinates are used to include GP effects $[18,6,8,9]$. Note that in general $f(\mathbf{Q})$ will be a function of all the nuclear degrees of freedom [15].

Finally, it has been shown [3] that in the BO approximation, the leading term of the nonadiabatic coupling assumes in the vicinity of the conical intersection the form

$\hat{C}_{+-}=G_{+-}+2 F_{+-} \nabla=\frac{1}{\rho^{2}} \frac{\partial}{\partial \varphi}$.

Thus, it can be neglected only if the nuclear wavefunctions depend on $\rho$ through a power of $v$ greater than $1 / 2$. This is due to the volume element which makes the integrand of the derivative coupling terms vary as $\propto \rho^{2 v+1}$. Thus, the condition $2 v+1>2$ must be satisfied in order to neglect such coupling terms. As it will be shown later, such a requirement can be satisfied in most cases. Considering now $G_{++}$and $G_{--}$in Eq. (5), the leading term of these diagonal matrix elements has been shown [3] to assume in the vicinity of the conical intersection the form

$G_{++}=G_{--}=-\frac{1}{4 \rho^{2}}$.

Based on the above considerations, the coupled differential equations in Eq. (3) can be reduced near the crossing seam to two independent equations referring to the upper and lower sheets separately, namely

$$
\left\{-\frac{\hbar^{2}}{2 \mu}\left(\nabla^{2}+G_{--}\right)+V_{-}\right\} \Psi_{-}=E_{-} \Psi_{-}
$$

for the lower sheet, with a similar equation in which the subscripts - are replaced by + being applicable to the upper sheet; $V_{-}$is the adiabatic PES for the lower sheet, and $\Psi_{-}$and $E_{-}$the vibrational wavefunction and corresponding vibrational energy, respectively (correspondingly for $V_{+}, \Psi_{+}$, and $E_{+}$). Given their similar forms, we can omit subscripts in the following discussion, which means that the discussion is valid both for the upper and lower adiabatic PESs.

\section{Nuclear wavefunctions near the conical inter- section}

In the 3D space spanned by such a system of spherical coordinates, the Hamiltonian assumes the form

$$
\begin{aligned}
\hat{H}_{v}= & -\frac{\hbar^{2}}{2 \mu}\left\{\frac{1}{\rho^{2}} \frac{\partial}{\partial \rho}\left(\rho^{2} \frac{\partial}{\partial \rho}\right)\right. \\
& +\frac{1}{\rho^{2}} \frac{1}{\sin \theta} \frac{\partial}{\partial \theta}\left(\sin \theta \frac{\partial}{\partial \theta}\right) \\
& +\frac{1}{\rho^{2}} \frac{1}{\sin ^{2} \theta} \frac{\partial^{2}}{\partial \varphi^{2}} \\
& \left.-\frac{1}{4 \rho^{2}}-\mathscr{V}(\rho, \theta, \varphi),\right\}
\end{aligned}
$$

where we have used $\mathscr{V}(\rho, \theta, \varphi)=\frac{2 \mu}{\hbar^{2}} V(\rho, \theta, \varphi)$. Thus, the vibrational wave equation, Eq. (21) or its equivalent for the upper sheet, will be given by

$$
\begin{gathered}
\left\{\frac{1}{\rho^{2}} \frac{\partial}{\partial \rho}\left(\rho^{2} \frac{\partial}{\partial \rho}\right)+\frac{1}{\rho^{2}} \frac{1}{\sin \theta} \frac{\partial}{\partial \theta}\left(\sin \theta \frac{\partial}{\partial \theta}\right)\right. \\
+\frac{1}{\rho^{2}} \frac{1}{\sin ^{2} \theta} \frac{\partial^{2}}{\partial \varphi^{2}}+\left[\frac{2 \mu E}{\hbar^{2}}-\frac{1}{4 \rho^{2}}\right. \\
-\mathscr{V}(\rho, \theta, \varphi)]\} \Psi_{v}(\rho, \theta, \varphi)=0 .
\end{gathered}
$$

Moreover, in the vicinity of the conical intersection, the potential energy can be shown [3] to assume a separable form with its leading term depending on $\rho$ alone. Separation of variables can then be obtained by writing the nuclear vibrational wavefunction as

$\Psi_{v}=R(\rho) \Theta(\theta) \Phi(\varphi)$,

which upon insertion into Eq. (23) leads to

$$
\begin{aligned}
& \left\{\frac{d^{2}}{d^{2} \varphi}+m^{2}\right\} \Phi(\varphi)=0, \\
& \left\{\frac{1}{\sin \theta} \frac{d}{d \theta}\left(\sin \theta \frac{d}{d \theta}\right)+\left[\lambda-\frac{m^{2}}{\sin ^{2} \theta}\right]\right\} \Theta(\theta)=0,
\end{aligned}
$$

$$
\begin{aligned}
& \left\{\frac{1}{\rho^{2}} \frac{d}{d \rho}\left(\rho^{2} \frac{d}{d \rho}\right)\right. \\
& \left.-\left[\varepsilon+\mathscr{V}(\rho)+\frac{\lambda}{\rho^{2}}+\frac{1}{4 \rho^{2}}\right]\right\} R(\rho)=0,
\end{aligned}
$$


where $\varepsilon=-(2 \mu E) / \hbar^{2}$, and we have considered the bound vibrational states to be defined such that $\varepsilon>0$. Regarding Eq. (25), the solutions clearly assume the form

$\Phi(\varphi)=(2 \pi)^{-1 / 2} \exp (\mathrm{i} m \varphi)$

where $m=n+(j / 2), n=0, \pm 1, \pm 2, \cdots$, and $j=$ 1 or 0 depending on whether one takes GP effects into consideration (GP case) or not (NGP case) [8]. Note that the quantum number $m$ has a meaning similar to $n_{\varphi}$ in Ref. [1] where the formalism has been developed in terms of hyperspherical coordinates [6].

Now considering Eq. (26), and using $\xi=\cos \theta$ $(-1 \leqslant \xi \leqslant 1)$, one gets

$$
\begin{aligned}
& {\left[\left(1-\xi^{2}\right) \frac{d^{2}}{d \xi^{2}}-2 \xi \frac{d}{d \xi}\right.} \\
& \left.+\left(\lambda-\frac{m^{2}}{1-\xi^{2}}\right)\right] \Theta(\theta)=0 .
\end{aligned}
$$

Assuming further that the solutions have the form

$$
\Theta(\xi)=\left(1-\xi^{2}\right)^{(|m| / 2)} v(\xi)
$$

where

$v(\xi)=\sum_{\nu=0}^{\infty} c_{\nu} \xi^{\nu}$,

one obtains, from Eqs. (29) and (30),

$$
\begin{gathered}
\left\{\left(1-\xi^{2}\right) \frac{d^{2}}{d \xi^{2}}-2(|m|+1) \xi \frac{d}{d \xi}\right. \\
\left.+\left(\lambda-|m|-m^{2}\right)\right\} v(\xi)=0 .
\end{gathered}
$$

Substitution of Eq. (31) into Eq. (32) then leads to

$$
\begin{aligned}
& \sum_{\nu=0}^{\infty}\left\{(\nu+2)(\nu+1) c_{\nu+2}-[\nu(\nu-1)\right. \\
& \left.\left.\quad+2(|m|+1) \nu+|m|-\lambda+m^{2}\right] c_{\nu}\right\} \xi^{\nu}=0,
\end{aligned}
$$

which implies

$$
\begin{aligned}
& (\nu+2)(\nu+1) c_{\nu+2} \\
& \quad=\left[\nu(\nu-1)+2(|m|+1) \nu+|m|-\lambda+m^{2}\right] c_{\nu} .
\end{aligned}
$$

Assuming next that the polynomials break-off at $c_{k} \xi^{k}$ (i.e., $c_{k+2}=0$ ), where $k$ is an integer or zero, one has

$k(k-1)+2(|m|+1) k+|m|-\lambda+m^{2}=0$

or

$\lambda=(k+|m|)(k+|m|+1)$.

Setting now $l=k+|m|$ as an integer, which includes zero in the NGP case, or a half-integer in the GP case, one obtains

$\lambda=l(l+1)$.

Thus, we may think of $l$ as an orbital angular momentum quantum number; note that $l$ has a meaning similar to $n_{\theta}$ in Ref. [1]. Furthermore, Eq. (29) is seen to be the Legendre equation, which has the well-established associated Legendre polynomials $P_{l}^{|m|}(\cos \theta)$ as solutions.

We now turn to solutions of the radial Schrödinger equation, Eq. (27). From this and Eq. (37), one gets

$$
\begin{aligned}
& \left\{\frac{1}{\rho^{2}} \frac{d}{d \rho}\left(\rho^{2} \frac{d}{d \rho}\right)\right. \\
& \left.-\left[\varepsilon+\mathscr{V}(\rho)+\frac{l(l+1)}{\rho^{2}}+\frac{1}{4 \rho^{2}}\right]\right\} R(\rho) \\
& \quad=0 .
\end{aligned}
$$

Assume then that the potential has in the vicinity of the conical intersection the general form

$\mathscr{V}(\rho)=\sum_{i=1}^{k} c_{i} \rho^{i}$

where for convenience we have chosen the reference $c_{0}=0$, and $c_{2}=1$ (note that such a form is applicable both to the upper and lower sheets of the PES after appropriate selection of the sign of $c_{1}$ ). Note also that, for large perimeters associated with equilateral triangle molecular shapes, one must satisfy the requirement

$\lim _{\rho \rightarrow \infty} \mathscr{V}(\rho) \rightarrow 0$ 
Thus, when $\rho \rightarrow \infty$, Eq. (38) assumes the form

$\left(\frac{d^{2}}{d \rho^{2}}-\varepsilon\right) R(\rho)=0$,

and hence $\lim _{\rho \rightarrow \infty} R(\rho) \rightarrow \exp ( \pm \sqrt{\varepsilon} \rho)$. As a result, the well-behaved asymptotic solution will be given by

$$
\lim _{\rho \rightarrow \infty} R(\rho) \rightarrow \exp (-\sqrt{\varepsilon} \rho)
$$

Clearly, the nuclear wavefunction decays quicker with increasing energy $(|E|)$ for bound vibrational states. For resonance states, $E$ will take complex values and hence the decay in Eq. (42) will display an oscillatory behavior. In turn, for scattering states one has $E>0$, and hence the nuclear wavefunction will oscillate.

In the limit of $\rho \rightarrow 0$, Eq. (38) assumes the form

$\left[\frac{d^{2}}{d \rho^{2}}+\frac{2}{\rho} \frac{d}{d \rho}-\frac{l(l+1)}{\rho^{2}}-\frac{1}{4 \rho^{2}}\right] R(\rho)=0$

since $\mathscr{V}(\rho) \rightarrow 0$. Eq. (43) has, in the limit of $\rho \rightarrow 0$, a behavior identical to the Bessel equation

$\left[\frac{d^{2}}{d \rho^{2}}+\frac{1}{\rho} \frac{d}{d \rho}+\left(1-\frac{v^{2}}{\rho^{2}}\right)\right] R(\rho)=0$.

Thus, it has the asymptotic solutions

$$
\lim _{\rho \rightarrow 0} R(\rho) \rightarrow \frac{\rho^{v}}{2^{v} \Gamma(v+1)}
$$

where

$v=\sqrt{l(l+1)+\frac{1}{4}}$.

Thus, for the NGP case, $v=\frac{1}{2}, \frac{3}{2}, \frac{5}{2}, \cdots$ corresponding to $l=0,1,2, \cdots$. In turn for the GP case, one has $v=1,2,3, \cdots$ corresponding to $l=\frac{1}{2}, \frac{3}{2}, \frac{5}{2}, \cdots$. Thus, all values of $v$ are greater than $1 / 2$ except for $v=1 / 2$ in the NGP case. The off-diagonal coupling term can therefore be removed as pointed out in Section 2. For $l=0$, we must therefore consider such coupling term. Note that the quantum number $v$ has now a meaning similar to $n_{\rho}$ in Ref. [1].
The general solution of Eq. (38) is then up to a constant factor, given by

$R(\rho)=\exp (-\sqrt{\varepsilon} \rho) \rho^{v} F(\rho)$

where, upon substitution of Eq. (47) in Eq. (38), $F(\rho)$ may be shown to satisfy

$$
\begin{aligned}
& \left\{\frac{d^{2}}{d \rho^{2}}+\left[\frac{2(v+1)}{\rho}-2 \sqrt{\varepsilon}\right] \frac{d}{d \rho}\right. \\
& \left.-\left[\frac{2(v+1) \sqrt{\varepsilon}}{\rho}+\mathscr{V}(\rho)\right]\right\} F(\rho)=0 .
\end{aligned}
$$

Consider then the asymptotic behavior of $F(\rho)$. In the vicinity of the conical intersection $\rho \rightarrow 0$, and hence Eq. (48) assumes the form

$\left[\frac{d^{2}}{d \rho^{2}}+\frac{2(v+1)}{\rho} \frac{d}{d \rho}-\frac{2(v+1) \sqrt{\varepsilon}}{\rho}\right] F(\rho)=0$,

which has the asymptotic solutions

$$
\lim _{\rho \rightarrow 0} F(\rho) \rightarrow{ }_{1} F_{1}(\gamma \sqrt{\varepsilon}, \gamma, \rho)
$$

where ${ }_{1} F_{1}(\gamma \sqrt{\varepsilon}, \gamma, \rho)$ is the confluent hypergeometric function (Kummer's function) with $\gamma=2(v+1)$. In turn, for $\rho \rightarrow \infty$, Eq. (48) takes the form

$$
\left[\frac{d^{2}}{d \rho^{2}}-2 \sqrt{\varepsilon} \frac{d}{d \rho}-\mathscr{V}(\rho)\right] F(\rho)=0 \text {. }
$$

If one now assumes that

$$
\lim _{\rho \rightarrow \infty} \mathscr{V}(\rho) \rightarrow \delta_{+}>0
$$

where $\delta_{+}$is taken as a sufficiently small positive number, the asymptotic solutions of $F(\rho)$ will assume the form

$$
\lim _{\rho \rightarrow \infty} F(\rho) \rightarrow \exp \left[\left(\sqrt{\varepsilon}-\sqrt{\varepsilon+\delta_{+}}\right) \rho\right] .
$$

Thus, $F(\rho)$ approaches unity both in the limits $\rho \rightarrow 0$ and $\rho \rightarrow \infty$.

For the GP case, the nuclear wavefunction assumes therefore the form

$$
\begin{aligned}
& \Psi(\rho, \theta, \varphi) \\
& \quad=N \exp (-\sqrt{\varepsilon} \rho) \rho^{v} F(\rho) P_{l}^{|m|}(\cos \theta) \exp (\operatorname{i} m \varphi)
\end{aligned}
$$


with

$l=k+\frac{1}{2}, k+\frac{3}{2}, \cdots \quad m= \pm \frac{1}{2}, \pm \frac{3}{2}, \cdots$.

In turn, for the NGP case, one has

$l=k+0, k+1, k+2, \cdots \quad m=0, \pm 1, \cdots$

where, as usual, $N$ is a normalization factor. Thus, since in the GP case $l$ is a half-integer and $v$ is a positive interger, one gets

$\lim _{\rho \rightarrow 0} \Psi(\rho, \theta, \varphi) \rightarrow 0$.

Similarly, for the NGP, one obtains Eq. (57). In these cases, the centrifugal potential including that due to $G_{++}$or $G_{--}$terms prevents the nuclear wavefunction from penetrating in the vicinity of the conical intersection. In fact, this appears to be the case for the ground state of $\mathrm{Li}_{3}$, as shown in Fig. 2 and Fig. 3; these illustrate the corresponding NGP and GP nuclear probability densities $|\Psi|^{2}$, which have been calculated using hyperspherical coordinates [8]. Specifically, they show the four lowest vibrational states which are labeled by their permutational symmetries $\mathrm{A}_{1}, \mathrm{~A}_{2}$ and $\mathrm{E}$ in $\mathscr{S}_{3}$ permutation group and by the set of vibrational quantum numbers $\left(v_{1}, v_{2}, v_{3}\right)$ in the $C_{2 v}$ point group, where $v_{1}, v_{2}$, and $v_{3}$ are associated to the symmetric stretching, bending, and asymmetric stretching vibrational modes [8]. Note that the conical intersection is located at the centre of the plot. Clearly, the nuclear wavefunctions approach zero at the conical intersection in all cases. Although in the NGP case with $l=0$ there is not an intrinsic centrifugal potential barrier, the potential due to $G_{++}$or $G_{--}$will play that role by prevent- (a)

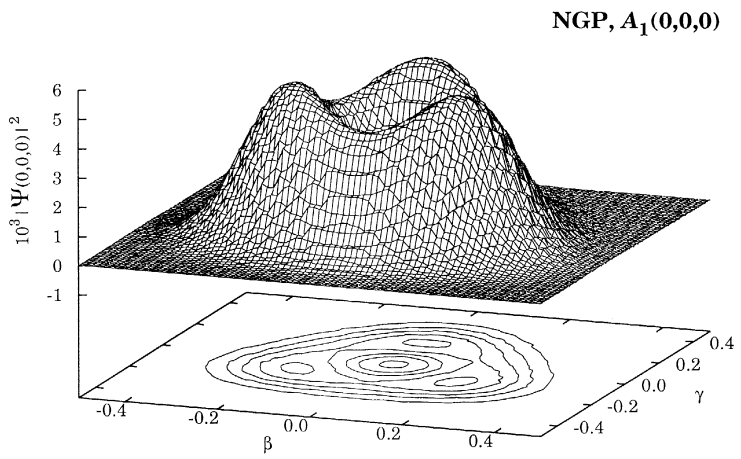

(c)

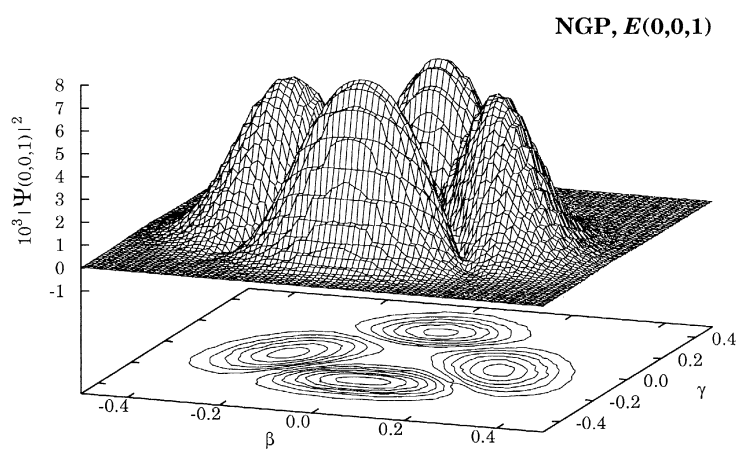

(b)

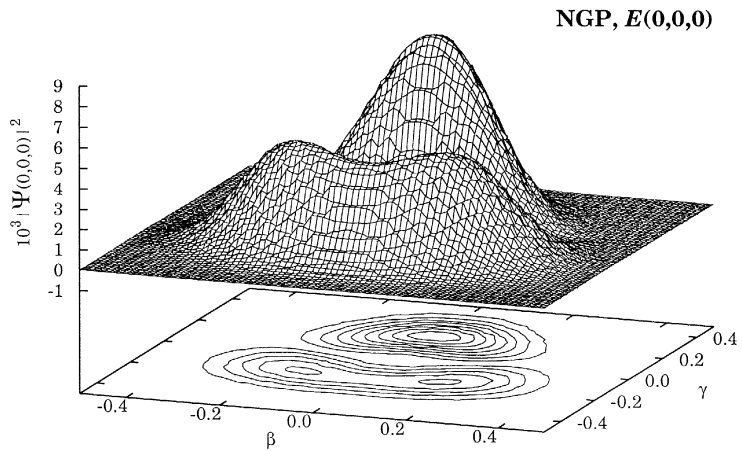

(d)

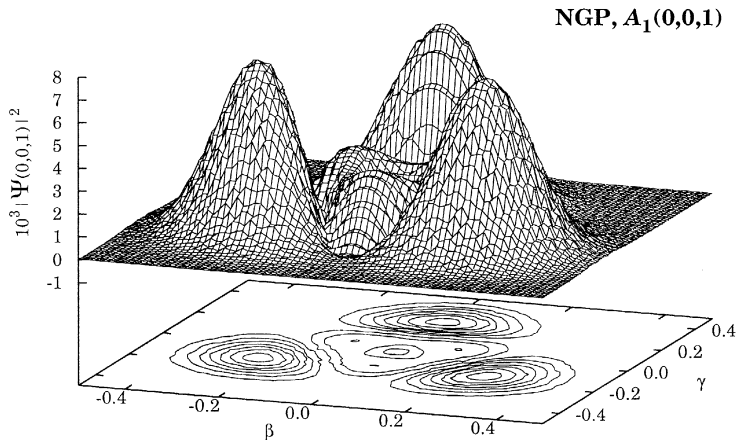

Fig. 2. Triangular plot [29] (perspective view/contour diagram) using hyperspherical coordinates of cross-sections for the nuclear probability densities without including GP effects. They refer to a value of Ref. [29] $Q=r_{\mathrm{AB}}^{2}+r_{\mathrm{BC}}^{2}+r_{\mathrm{AC}}^{2}=91.74 a_{0}^{2}$, which corresponds to a hyperradius for which the density probability, after averaging over the hyperangles $\theta$ and $\phi$, is maximum; $\beta=\beta^{\star} Q$ and $\gamma=\gamma^{\star} Q$, and $\mathrm{A}, \mathrm{B}$, and $\mathrm{C}$ denote the atoms (1, 2, and 3 in Fig. 1). Note that the conical intersection is located at the centre of the plot. Key for panels: (a) $\mathrm{A}_{1}(0,0,0)$; (b) one component of the $\mathrm{E}(0,0,0)$ state; (c) one component of $\mathrm{E}(0,0,1)$; (d) $\mathrm{A}_{1}(0,0,1)$. 
(a)

(b)

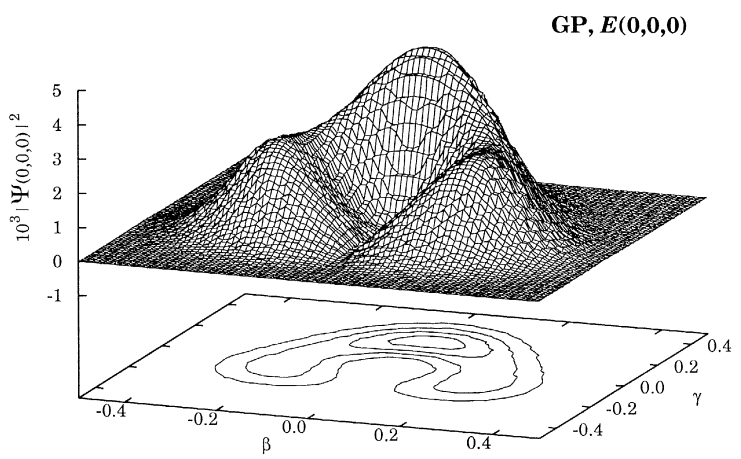

(c)

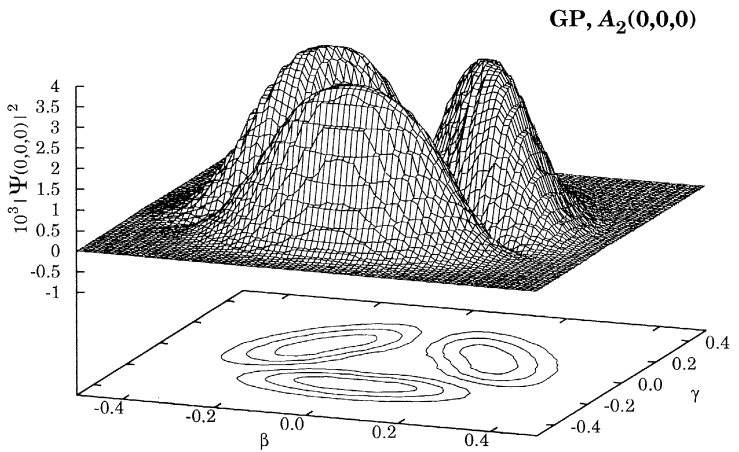

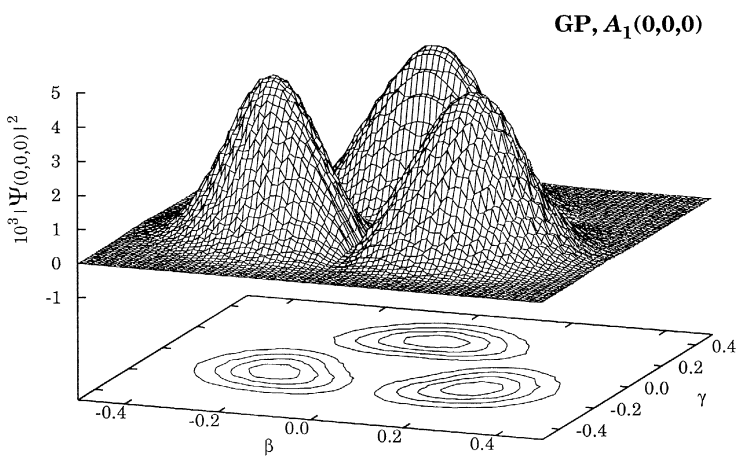

(d)

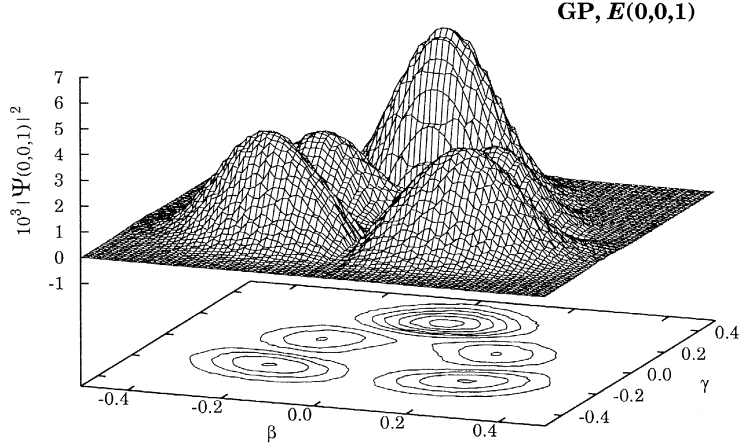

Fig. 3. As in Fig. 2 but with inclusion of GP effects. Key for panels: (a) one component of $E(0,0,0) ;(b) A_{1}(0,0,0) ;\left(\right.$ c) $A_{2}(0,0,0) ;(d)$ one component of $E(0,0,1)$.

ing the nuclear wavefunction from penetrating in the vicinity of the conical intersection. However, as we have mentioned before, the coupling terms corresponding to the operator $\hat{C}_{+-}$have a non-zero value in this case, and hence a two-state calculation must be considered.

To conclude, our results may be compared with the approximate 2D solutions reported by Mead [2],

Table 1

Comparison of the first lowest values of $\left(m^{\prime}, v^{\prime}\right)$ in Eq. (58) and $(m, l, v)$ in Eq. (54)

\begin{tabular}{|c|c|c|c|c|}
\hline \multicolumn{2}{|l|}{ Mead [2] } & \multicolumn{3}{|l|}{ This work } \\
\hline$\overline{m^{\prime}}$ & $v^{\prime}$ & $\bar{m}$ & $l$ & $\bar{v}$ \\
\hline$-1,-2$ & $\sqrt{2} / 2=0.707$ & $\pm 1 / 2$ & $1 / 2$ & 1 \\
\hline $0,-3$ & $\sqrt{10} / 2=1.581$ & $\pm 1 / 2, \pm 3 / 2$ & $3 / 2$ & 2 \\
\hline $1,-4$ & $\sqrt{26} / 2=2.550$ & $\pm 1 / 2, \pm 3 / 2, \pm 5 / 2$ & $5 / 2$ & 3 \\
\hline $2,-5$ & $\sqrt{50} / 2=3.536$ & $\pm 1 / 2, \pm 3 / 2, \pm 5 / 2, \pm 7 / 2$ & $7 / 2$ & 4 \\
\hline $3,-6$ & $\sqrt{82} / 2=4.528$ & $\pm 1 / 2, \pm 3 / 2, \pm 5 / 2, \pm 7 / 2, \pm 9 / 2$ & $9 / 2$ & 5 \\
\hline
\end{tabular}


$\Psi(\rho, \varphi)=\rho^{v^{\prime}} \exp \left(\mathrm{i} m^{\prime} \varphi\right)$

where

$v^{\prime}=\sqrt{\left(m^{\prime}+\frac{3}{2}\right)^{2}+\frac{1}{4}} \quad m^{\prime}=0, \pm 1, \pm 2 \cdots$.

Clearly, Mead's asymptotic wavefunction approaches zero slower than ours probably due to having considered only a 2D treatment (note also that it does not change sign when $\varphi \rightarrow \varphi+2 \pi)$. This is illustrated by the values given in Table 1 . Indeed, the rates of decrease differ marginally from Mead's ones, especially for large values of $v^{\prime}$ (denoted $n$ in Ref. [2]) where these approach our values of $v$. It seems therefore that the quantum numbers are exact only in the $3 \mathrm{D}$ treatment of the present work.

\section{Closing remarks}

We have analyzed the behavior of the nuclear wavefunction in the vicinity of the conical intersection which arises at equilateral triangle configurations for homonuclear triatomic systems. It has been found that, in the vicinity of the equilibrium configuration, the motion of the nuclei can be described by the quantum numbers $n_{\theta}=l$ and $n_{\varphi}=m$ and also by the quantum number $n_{\rho}=v$. The set of quantum numbers $\left(n_{\theta}, n_{\varphi}\right)$ has been shown to take half-integer values in the GP case while assuming integer or zero values for the NGP one. Moreover, the quantum number $n_{\rho}$ has been shown to assume integer values in the GP case while being half-integer in the NGP case. Since our derivation is more general than the one given by Mead [2], we have obtained a set of exact quantum numbers to quantize the motion in such regions while in his case the quantum number associated to the $\rho$ coordinate is only approximate. It has also been shown that the nuclear wavefunctions approach zero, both in the NGP and GP cases.

\section{Acknowledgements}

This work has been supported by the Fundação para a Ciência e Tecnologia, Portugal, under pro- grammes PRAXIS XXI and and FEDER (Contract No. 2/2.1/QUI/408/94).

\section{References}

[1] A.J.C. Varandas, Z.R. Xu, in: R. Bader, L. Montero (Eds.), Topics of Current Computational Chemistry, Kluwer, Dordrecht, 1999, in press.

[2] C.A. Mead, J. Chem. Phys. 78 (1983) 807.

[3] T.C. Thompson, D.G. Truhlar, C.A. Mead, J. Chem. Phys. 82 (1985) 2392.

[4] T.C. Thompson, C.A. Mead, J. Chem. Phys. 82 (1985) 2408.

[5] A. Kuppermann, Y.-S.M. Wu, Chem. Phys. Lett. 241 (1995) 229.

[6] A.J.C. Varandas, H.G. Yu, J. Chem. Soc. Faraday Trans. 93 (1997) 819.

[7] W.H. Gerber, E. Schumacher, J. Chem. Phys. 69 (1978) 1692.

[8] A.J.C. Varandas, H.G. Yu, Z.R. Xu, Mol. Phys. 96 (1999) 1193.

[9] A.J.C. Varandas, Z.R. Xu, Int. J. Quantum Chem. 75 (1999) 89.

[10] B. Kendrick, Phys. Rev. Lett. 79 (1997) 2431.

[11] G. Herzberg, H.C. Longuet-Higgins, Faraday Discuss. Chem. Soc. 35 (1963) 77.

[12] M.V. Berry, Proc. R. Soc. A 392 (1984) 45.

[13] H.C. Longuet-Higgins, Proc. R. Soc. London A 344 (1975) 147.

[14] A.J.C. Varandas, J. Tennyson, J.N. Murrell, Chem. Phys. Lett. 61 (1979) 431.

[15] B. Kendrick, R. Pack, J. Chem. Phys. 104 (1996) 7475.

[16] M. Baer, R. Englman, Mol. Phys. 75 (1992) 293.

[17] M. Baer, Chem. Phys. Lett. 265 (1997) 105.

[18] G.D. Billing, N. Marković, J. Chem. Phys. 99 (1993) 2674.

[19] J.E.B. Wilson, J.C. Decius, P.C. Cross, Molecular Vibrations: the Theory of Infrared and Raman Spectra, McGrawHill, New York, 1955.

[20] S. Califano, Vibrational States, Wiley, London, 1976.

[21] M. Baer, Chem. Phys. Lett. 35 (1975) 112.

[22] C.A. Mead, D.G. Truhlar, J. Chem. Phys. 77 (1982) 6090.

[23] D.R. Yarkony, J. Chem. Phys. 105 (1996) 10456.

[24] N. Matsunaga, D.R. Yarkony, J. Chem. Phys. 107 (1997) 7825.

[25] R.G. Sadygov, D.R. Yarkony, J. Chem. Phys. 109 (1998) 20.

[26] H.C. Longuet-Higgins, Adv. Spectrosc. 2 (1961) 429.

[27] C.A. Mead, D.G. Truhlar, J. Chem. Phys. 70 (1979) 2284.

[28] C.A. Mead, Chem. Phys. 49 (1980) 23.

[29] A.J.C. Varandas, Chem. Phys. Lett. 138 (1987) 455. 